\title{
Emerging role for SRC family kinases in junction dynamics during spermatogenesis
}

\author{
Xiang Xiao ${ }^{1}$, Yue Yang ${ }^{1}$, Baiping $\mathrm{MaO}^{2}, \mathrm{C}$ Yan $\mathrm{Cheng}^{2}$ and $\mathrm{Ya} \mathrm{Ni}^{1}$ \\ ${ }^{1}$ Department of Reproductive Physiology, Zhejiang Academy of Medical Sciences, Hangzhou, Zhejiang, China and \\ ${ }^{2}$ The Mary M. Woldford Laboratory for Male Contraceptive Research, Center for Biomedical Research, Population \\ Council, New York, New York, USA
}

Correspondence should be addressed to CY Cheng or Y Ni; Email: Y-Cheng@popcbr.rockefeller.edu or niya99@126.com

\begin{abstract}
SRC family kinases (SFKs) are known regulators of multiple cellular events, including cell movement, differentiation, proliferation, survival and apoptosis. SFKs are expressed virtually by all mammalian cells. They are non-receptor protein kinases that phosphorylate a variety of cellular proteins on tyrosine, leading to the activation of protein targets in response to environmental stimuli. Among SFKs, SRC, YES and FYN are the ubiquitously expressed and best studied members. In fact, SRC, the prototypical SFK, was the first tyrosine kinase identified in mammalian cells. Studies have shown that SFKs are regulators of cell junctions, and function in endocytosis and membrane trafficking to regulate junction restructuring events. Herein, we briefly summarize the recent findings in the field regarding the role of SFKs in the testis in regulating spermatogenesis, particularly in Sertoli-Sertoli and Sertoli-germ cell adhesion. While it is almost 50 years since the identification of the oncogene v-Src encoded by Rous sarcoma transforming virus, the understanding of SFK involvement during spermatogenesis in the testis remains far behind that in other epithelia and tissues. The goal of this review is to bridge this gap.

Reproduction (2019) 157 R85-R94
\end{abstract}

\section{Introduction}

During spermatogenesis, type A spermatogonia undergo either mitotic proliferation for self-renewal or differentiation into type B spermatogonia by 8 and $6 \mathrm{dpp}$ in mouse and rat testes, respectively (Clermont \& Perry 1957, Bellve et al. 1977). These germ cell types reside behind the blood-testis barrier (BTB) at the basal compartment of the seminiferous epithelium (Fig. 1). Type B spermatogonia subsequently give rise to preleptotene and then leptotene spermatocytes, which are the only germ cell types to be transported across the BTB with the aid from Sertoli cells in rodents (de Kretser \& Kerr 1988, Smith \& Braun 2012). This reliance on Sertoli cells for their transport across the BTB is necessary since the germ cells do not possess either filopodia or lamellipodia found in other motile cells such as macrophages, fibroblasts or neutrophils (Cheng \& Mruk 2002). The developing germ cells enter the adluminal compartment to further differentiate and transform into zygotene, pachytene and diplotene spermatocytes to enter meiosis I/II (Clermont 1972, Parvinen 1982, Hess \& de Franca 2008, Hermo et al. 2010a). Thereafter, haploid cells (step 1, round spermatids) derived from meiosis undergo spermiogenesis, which consists of extensive morphological changes, to form elongating and elongated spermatids via 16 and 19 steps in mouse and rat testes, respectively (Hess \& de Franca 2008, Xiao et al. 2014a). Eventually, sperm line up near the tubule lumen to prepare for their release at spermiation during stage VII-VIII of the epithelial cycle. After spermiation, testicular sperm enter the epididymis so that they can undergo post-testicular maturation to become fully functional sperm, capable of undergoing capacitation and acrosome reaction to fertilize the egg in the female reproductive tract.

It is envisioned that spermatid transport by Sertoli cells across the seminiferous epithelium in the adluminal compartment is one of the crucial cellular events to support spermatogenesis, in particular, spermiogenesis because without the timely transport, spermiation fails, and infertility occurs. But the biology underlying spermatid transport remains unclear (Vogl et al. 2008, 2013, Hermo et al. 2010b, O'Donnell et al. 2011, Xiao et al. 2014a, Cheng \& Mruk 2015, Tang et al. 2016). Recent studies have shown that this cellular event relies on the support of F-actin- and microtubule (MT)based cytoskeletons, likely involving specific regulatory proteins modulating biogenesis, organization, polymerization and depolymerization of cytoskeletal networks (O'Donnell \& O'Bryan 2014, Wen et al. 2016). An actin-based testis-specific cell-cell anchoring junction ectoplasmic specialization (ES) is present at the interface of elongating/elongated spermatids 


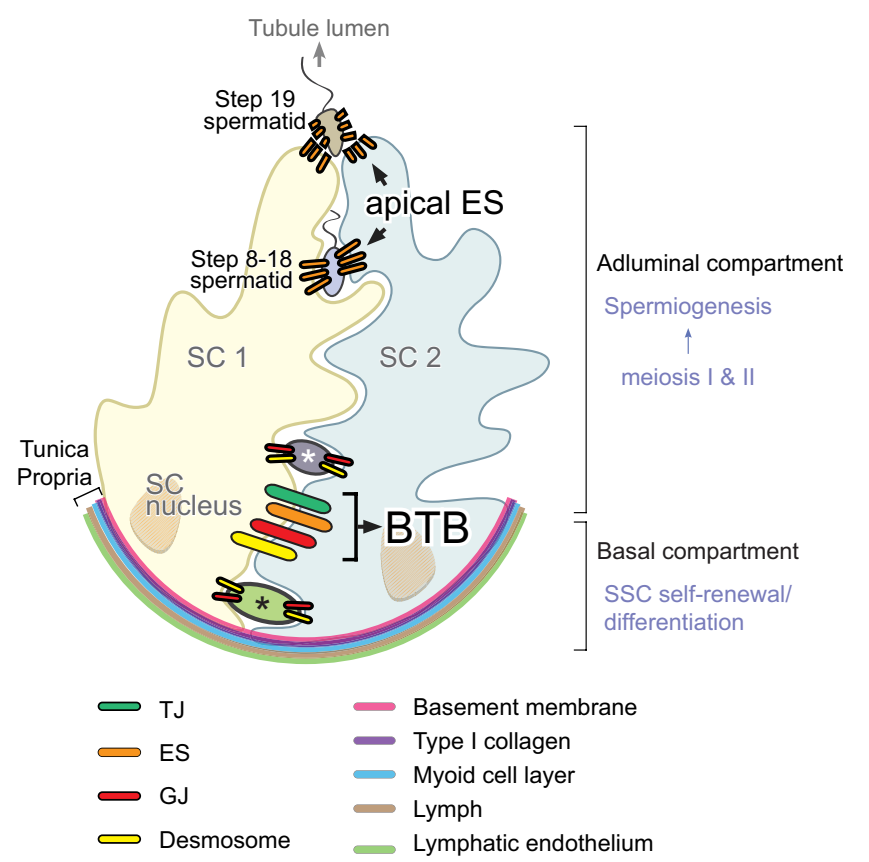

Figure $1 \mathrm{~A}$ schematic diagram illustrating the relative localization of the BTB and different junction types at the Sertoli cell-cell and Sertoli-germ cell interface in adult rat testes to support spermatogenesis (Sertoli and germ cell arrangement at a specific stage is omitted). The blood-testis barrier (BTB) divides the seminiferous epithelium into the adluminal and basal compartments, which are composed of only Sertoli and germ cells at different stages of their development, lying above the tunica propria. The BTB is constituted by coexisting actin-based tight junction (TJ), basal ectoplasmic specialization (ES) and gap junction (GJ), as well as intermediate filament-based desmosome. During spermatogenesis, spermatogonia, primary spermatocytes (i.e., preleptotene/leptotene spermatocytes) residing in the basal compartment (annotated with a black asterisk), and more-developed primary spermatocytes (i.e., zygotene, pachytene and diplotene spermatocytes), secondary spermatocytes and step 1-7 spermatids residing in the adluminal compartment (annotated with a white asterisk), are supported by desmosome and gap junction at the Sertoli-germ cell interface. Apical ES first appears at the interface of step 8 spermatid-Sertoli cell in stage VIII tubules, replacing desmosome and gap junction as the only anchoring device until step 19 spermatids, which transform to become sperm to prepare for their release at spermiation in stage VII-VIII tubules when apical ES undergoes degeneration. The tunica propria is comprised of the basement membrane, the type I collagen layer, myoid cell layer, lymph and endothelia cells of the lymphatic vessel.

(step 8-19 vs $8-16$ spermatids in the rat and mouse testis, respectively) and Sertoli cells in the adluminal compartment, which is designated as apical ES. Once apical ES appears in step 8 spermatids at stage VIII of the epithelial cycle (Russell 1977b, de Kretser \& Kerr 1988, Xiao et al. 2014a), it is the only anchoring device at the site until spermiation. ES is also found between adjacent Sertoli cells at the BTB known as the basal ES (Russell \& Peterson 1985, Vogl et al. 2000, 2008, Cheng \& Mruk 2015). Different from apical ES, basal ES coexists with actin-based tight junction (TJ) and gap junction, which localize together with the intermediate filamentbased desmosome, and as a whole, they confer to the BTB integrity (Vogl et al. 2008, 2013, Pelletier 2011, Cheng \& Mruk 2012) (Fig. 1). At stages VII-VIII of the epithelial cycle in both mouse and rat testes, elongated spermatids in adluminal compartment are preparing for their release into the tubule lumen at spermiation, necessitating apical ES degeneration at the Sertoli cell-spermatid interface. In the meantime, BTB also undergoes extensive remodeling to accommodate the transit of preleptotene/leptotene spermatocytes (residing in the basal compartment) across the immunological barrier (de Kretser \& Kerr 1988, Hess \& de Franca 2008). Conceivably, these two synchronized processes during spermatogenesis involve substantial cytoskeletal rearrangement and turnover of cell junctions, such as ES at the apical ES and BTB/basal ES sites, which is known to support spermatid transport (Mruk \& Cheng 2004) and translocation of spermatocytes through the BTB (Russell 1977a). However, the molecules and/ or the mechanism(s) underlying these concerted and coordinated cellular events, and how apical ES and BTB morph cyclically to facilitate germ cell transport are left largely unrevealed. Studies during the past decade support the notion that SRC family kinases (SFKs) are active participants in spermatogenesis (Maekawa et al. 2002, Goupil et al. 2011, Xiao et al. 2012, 2014b, Chojnacka \& Mruk 2015, Mruk et al. 2017), and may play a role in BTB restructuring and disassembly of Sertoli cell-spermatid junctions. Herein, we focus primarily on the better studied SFKs, such as SRC and YES in spermatogenesis, and evaluate their involvement in cell junction dynamics, and how they influence cytoskeletal organization and endocytic vesicle-mediated protein trafficking events to support junction remodeling. We also provide a hypothetical model based on these studies that phosphorylation of cell adhesion protein by either SRC or YES serves as a hub to determine if the protein should be targeted to endosome-mediated protein degradation vs its recycling. This model could serve as a point of reference for future studies on BTB restructuring and SFK's role in regulating spermatid adhesion to Sertoli cells.

\section{Overview of SFKs and findings in testis}

As non-receptor protein tyrosine kinases, SFKs are known to phosphorylate large number of substrates on tyrosine, leading to the activation of protein targets in response to environmental stimuli; and many of the substrates are phosphorylated by multiple SFKs (Takeda et al. 2010, Espada \& Martin-Perez 2017), which suggests the complementarities of SFK functions. SFKs are widely involved in signaling events that regulate various epithelial functions, such as cell migration, cell proliferation and differentiation (e.g., neuronal differentiation), cell survival and apoptosis, calcium 
signaling, androgen signaling and spermatogenesis. Since the overexpression and/or hyperactivation of SFKs is commonly observed in pathogenesis of diseases, such as tumorigenesis (e.g., prostate cancer progression), HIV-1 infection and dementia (Engen et al. 2008, Xiao et al. 2012, Anguita \& Villalobo 2017, Castoria et al. 2017, Coiras et al. 2017, Espada \& Martin-Perez 2017, Kim et al. 2017, Nygaard 2018), specific SFK inhibitors have been used and/or investigated to treat various diseases including cancers and neurodegenerative diseases. In brief, the physiological role of SFKs on many other cellular functions has been the subject of several recent reviews (Xiao et al. 2012, Castoria et al. 2017, Coiras et al. 2017, Espada \& Martin-Perez 2017, Kim et al. 2017, Nygaard 2018).

There are at least eight SFK members, including SRC, YES, FYN, LYN, LCK, HCK, FGR and BLK, which are expressed in mammals. Among them, SRC, YES and FYN are the best studied and found in a variety of cell types, while the others are predominant in hematopoietic cells (Thomas \& Brugge 1997, Espada \& Martin-Perez 2017). Also, SRC, YES and FYN are the most ubiquitously expressed SFKs, able to compensate for the loss of one another since only triple knockouts show embryonic lethality (Stein et al. 1994). Emerging evidence has shown that SRC, YES and FYN are F-actin regulators in the testis, and may operate in cell adhesion at the Sertoli cell-cell and/or Sertoli cell-spermatid interface via their effect on actin-binding/regulatory proteins, such as Eps8 (epidermal growth factor receptor pathway substrate $8)$, an actin barbed end capping and bundling protein that confers actin filaments to a bundled configuration to maintain ES integrity in the seminiferous epithelium (Maekawa et al. 2002, O'Donnell et al. 2011, Cheng \& Mruk 2012, Xiao et al. 2013, Chojnacka \& Mruk 2015, Mruk et al. 2017). SRC and YES are expressed by both Sertoli and germ cells, and confirmed to be an integrated component of BTB and apical ES in the rat testis (Xiao et al. 2012, Chojnacka \& Mruk 2015). FYN has also been found to localize to ES and can phosphorylate and promote plakoglobin (an $\mathrm{ES} /$ desmosome protein) interaction with A-catenin (Maekawa et al. 2002, Mruk et al. 2017). Revealed by immunohistochemistry, RT-PCR or immunoblot analyses, the other SFKs have also been shown to be present in testis and likely involved in spermatogenesis (Kierszenbaum 2006, Lalancette et al. 2006, Bordeleau \& Leclerc 2008, Goupil et al. 2011, Xiao et al. 2012, Singh et al. 2017). Additionally, besides their full-length forms, some SFKs (e.g., FYN, HCK) are found in testis as truncated variants devoid of intact kinase domain, which probably function in spermiogenesis as adaptor proteins and/or competitive inhibitors independent from their kinase activity (Kierszenbaum 2006, Lalancette et al. 2006, Bordeleau \& Leclerc 2008, Kierszenbaum et al. 2009). Still, the exact functional details of different SFKs as well as their truncated forms in spermatogenesis are yet to be fully elucidated. It is of interest to note that studies have illustrated the role of SFKs in supporting drug transporter function, which is explicitly important regarding the BTB uptake of nutrients, drugs and even toxicants into the adluminal compartment of the seminiferous tubule (Klein \& Cherrington 2014). For instance, phosphorylation by YES is crucial to support OCT (organic cation transporter) activity in murine kidneys, such as OCT2 (Sprowl et al. 2016). By using SRC kinase inhibitor PP2, SFK has been shown required for a lysophosphatidic acid 1 receptor-mediated signaling cascade that regulates P-glycoprotein transport activity at the blood-brain barrier (Banks et al. 2018). Whether there is a similar function of SFKs at the BTB remains to be examined.

All SFKs share well-conserved structural domains across their polypeptide sequences as noted in Fig. 2 . Each member contains the following functional domains: (i) an N-terminal SRC homology (SH) 4 domain which contains the lipid modification sites, allowing for fatty acid acylation of SFK and subsequent linking to the plasma membrane; (ii) a non-conserved region 'unique' to each SFK member but with no clear function assigned, which, together with the $\mathrm{SH} 4$ domain, forms an N-terminal intrinsically disordered region (IDR) maintained in a compact yet dynamic state; (iii) an SH3 domain that binds to specific proline-rich sequences of partner proteins; (iv) an $\mathrm{SH} 2$ domain that binds to specific tyrosine phosphorylation sites of partner proteins; ( $v$ ) a
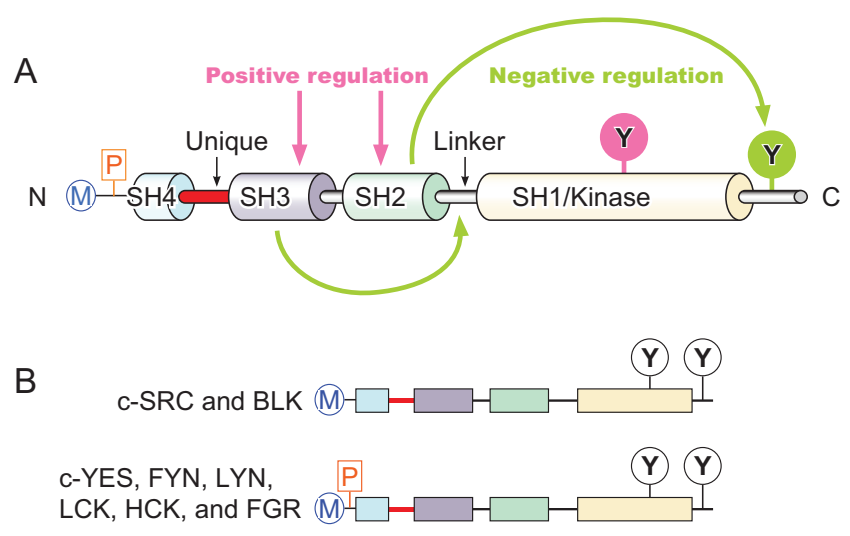

Figure 2 A schematic diagram illustrating the different functional and structural domains of members of the non-receptor SRC family kinases (SFKs). (A) This is the general layout of the different functional domains of SFKs. From the $\mathrm{N}$-terminal, there is a penultimate glycine (Gly2, i.e. the second amino acid from the $\mathrm{N}$-terminus) co-translationally myristoylated (M) which is used for SFK membrane targeting and to elicit signal transduction. A cysteine residue (Cys3, i.e. the third amino acid from the $\mathrm{N}$-terminus) of SFK is posttranslationally palmitoylated $(\mathrm{P})$, to be used for subcellular trafficking of SFK such as to the plasma membrane. This is followed by 4 SRC homology ( $\mathrm{SH}$ ) domains: $\mathrm{SH} 4, \mathrm{SH} 3$ and $\mathrm{SH} 2$ domains and then the $\mathrm{SH} 1 /$ kinase domain having the intrinsic kinase activity. (B) This illustrates the general layout of different SFK members, and the different colored domains correspond to the functional domains shown in (A). Y, Tyr. 
polyproline linker between $\mathrm{SH} 2$ and $\mathrm{SH} 1$ domains; (vi) an $\mathrm{SH} 1$ intrinsic tyrosine kinase catalytic domain and (vii) a negative regulatory tail region for auto-inhibition of kinase activity (Brown \& Cooper 1996, Thomas \& Brugge 1997, Arbesu et al. 2017). Under normal physiological conditions, SFK is inactive via autoinhibition in which $\mathrm{SH} 2$ domain binds to the inhibitory phosphotyrosine (p-Tyr, Tyr530 in SRC vs Tyr535 in YES) at the C-terminal tail, whereas $\mathrm{SH} 3$ domain interacts with polyproline linker between $\mathrm{SH} 2$ and $\mathrm{SH} 1$ domains, which thus blocks the $\mathrm{SH} 1 /$ intrinsic kinase domain by stabilizing SFK into an inactive conformation. SFKs can be activated following ligand binding to the $\mathrm{SH} 2$ and/ or $\mathrm{SH} 3$ domain or by dissociation of inhibitory $\mathrm{p}$-Tyr and unlatching as catalyzed by various protein tyrosine phosphatases. This in turn leads to a conformational change, allowing phosphorylation of the stimulatory Tyr in the SH1/kinase domain (Tyr419 in SRC vs Tyr424 in YES) to confer intrinsic kinase activity in the $\mathrm{SH} 1$ domain (Thomas \& Brugge 1997, Roskoski 2015, Espada \& Martin-Perez 2017). Although the three folded domains (SH3, SH2 and $\mathrm{SH} 1)$ are regulatory/catalytic domains responsible for the classical mechanism of SFK activity, recent studies have shown that the intrinsically unfolded domains ( $\mathrm{SH} 4$ and Unique), as well as their interactions with $\mathrm{SH} 3$ domain, may represent a new modulator of SFK functions (Perez et al. 2013, Amata et al. 2014, Arbesu et al. 2017).

As mentioned above, the $\mathrm{N}$-terminal acylation determines the plasma membrane targeting of SFKs. SFKs usually localize in cell cytosol close to plasma membrane through myristoylation and/or palmitoylation (Thomas \& Brugge 1997, Kasahara et al. 2004, Sato et al. 2009, Chu et al. 2014) (Fig. 2). Interestingly, the palmitoylation state in the $\mathrm{SH} 4$ domain of SFKs affects their subsequent cellular localization, thereby affecting the site(s) where a specific SFK exerts its regulatory function. For instance, YES is a mono-palmitoylated SFK, and it is transported through a Rab11-dependent pathway preferably from the Golgi pool of caveolin to the plasma membrane (Sato et al. 2009), implicating its possible role in supporting endocytosis, recycling and/ or transcytosis of integral membrane proteins, such as those localized at the apical and the basal ES in testis to support spermatogenesis. SRC, on the other hand, is a non-palmitoylated SFK, rapidly transported between the plasma membrane and late endosomes or lysosomes (Kasahara et al. 2007, Sato et al. 2009), illustrating the possibility that it may be involved in endosome-mediated protein degradation. While future studies are needed to confirm these possibilities, the observations are important since they demonstrate that, although highly homologous, YES and SRC can work divergently, and they likely function in concert to modulate intracellular protein trafficking events to support protein turnover and homeostasis (Xiao et al. 2012). Accordingly, studies have shown that by late stage VIII of the epithelial cycle in the rat testis, expression of YES increases at the BTB but decreases to an undetectable level at the apical ES around spermiation, whereas that of SRC goes the opposite (Lee \& Cheng 2005, Xiao et al. 2011, 2012, Chojnacka \& Mruk 2015), suggesting that these two SFKs can indeed play differential roles in junction dynamics/turnover at the BTB and apical ES sites, thus supporting germ cell transport and/or spermiation during the epithelial cycle of spermatogenesis (which we will discuss further below). Along this line, although how other SFKs (such as FYN which is dually palmitoylated) contribute to restructuring of the seminiferous epithelium has not been clearly defined, we suppose it might work differently with function(s) that cannot be substituted by dominantly expressed SRC. Consistent with this, when using cross-sections of adult rodent testes examined by immunohistochemistry, results have shown that there were differences in the localization patterns of SRC, YES and FYN in the seminiferous epithelium (especially from stage VII to late stage VIII tubules). For instance, unlike SRC or YES (Xiao et al. 2011, 2012, Chojnacka \& Mruk 2015), FYN expression in the seminiferous epithelium had its own distinct pattern that is maintained at a relatively stable level both at the apical ES and BTB/basal ES sites throughout spermatogenesis (Maekawa et al. 2002) (Fig. 3). Also, while they were all detected in the basal ES/BTB, YES was more prominent in these stages at the site, whereas at the apical ES, SRC and FYN were more prominent when compared with YES (Maekawa et al. 2002, Xiao et al. 2011, 2012, Chojnacka \& Mruk 2015). Furthermore, FYN was more extended that it appeared as track-like (or stalk-like) structures that expanded across the seminiferous epithelium (Maekawa et al. 2002) (Fig. 3). Collectively, these observations suggest that these SFKs may not be playing redundant functions in testis to modulate spermatogenesis. Instead, they may have different roles in supporting spermatogenesis.

\section{Differential roles of SFKs on endocytic protein trafficking in the testis}

During spermatogenesis, millions of germ cells at different developmental stages are formed along the entire length of the seminiferous tubules which are $\sim 26-\mathrm{m}$ in length, with a diameter at $\sim 280 \mu \mathrm{m}$, in rat testes at 1 year of age (Gaytan et al. 1986), illustrating considerable cellular activity in the tubules, including turnover of cell junctions. For instance, step 8 spermatids first appear at stage VIII of the epithelial cycle when apical ES must be actively assembled to support spermiogenesis at the Sertoli cell-spermatid interface (Vogl et al. 1993, 2008, Cheng \& Mruk 2010). Although the testis synthesizes different categories of proteins on a daily basis, due to the unusual cellular outputs to support spermatogenesis (e.g. the testis pair in rats and humans produce an upwards of $\sim 50-70$ to 

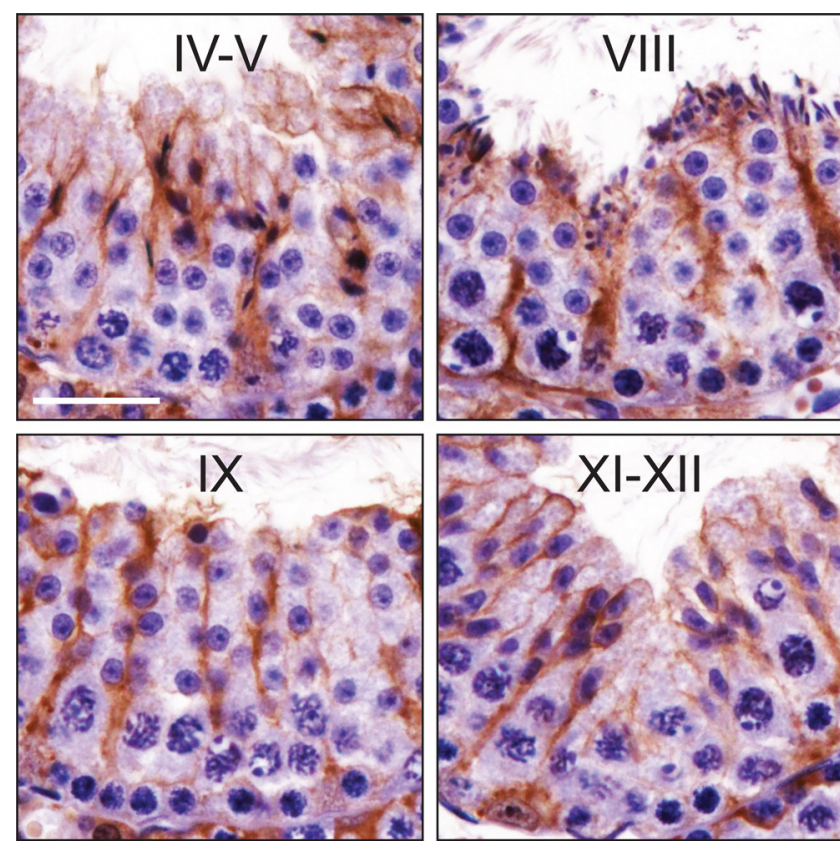

Figure 3 A study by immunohistochemistry to illustrate the localization of FYN in the seminiferous epithelium using crosssections of adult mouse testes. Immunohistochemistry was performed essentially as earlier described (Xiao et al. 2011), using a specific antibody against FYN (Santa Cruz, Cat \#:sc-16, dilution 1:100). FYN (brown precipitate) is present in all stages of the seminiferous epithelial cycle. Roman numerals indicate the stages of the seminiferous epithelial cycle. FYN is localized at the BTB and the apical ES, appearing as stalk-like structures extending across the seminiferous epithelium. These results agree with previously published reports (Maekawa et al. 2002). Scar bar, $30 \mu \mathrm{m}$, which apply to other corresponding micrographs.

>300 million sperm daily (Johnson et al. 1980, 1983), and given the fixed number of Sertoli cells following puberty in humans (at $\sim 12$ years of age) and rodents (at 17-19 days of age) to support germ cell development (Sharpe et al. 2003), it is physiologically impossible for de novo synthesis of all the required apical ES proteins (e.g., N-cadherin, B-catenin, nectin 2, nectin 3, afadin, laminins, A6 integrin, B1 integrin, JAM3 and others) vs basal ES proteins (e.g., $\mathrm{N}$-cadherin, B-catenin, nectin 2, afadin, occludin, ZO-1, JAM1, JAM2, claudins and others) (Cheng \& Mruk 2002). Instead, some of the proteins at the ES must be rapidly recycled to support protein turnover at the cell junctions during spermatogenesis. Corresponding to such mechanism, at late stage VII of the epithelial cycle, apical and basal ES undergo extensive remodeling to transform into a transient ultrastructure designated apical and basal TBC (tubulobulbar complex) respectively (Young et al. 2012b, Du et al. 2013, Vogl et al. 2013, 2014, Lyon et al. 2015), and proteins necessary to support endocytic vesicle-mediated protein trafficking, including clathrin, N-WASP, cortactin (Young et al. 2009a,b, 2012a), zyxin and vinculin (Young \& Vogl 2012), Arp3 (Lie et al. 2010),
Eps8 (Lie et al. 2009), and a newly identified $\mathrm{Ca}^{2+}$ signaling machinery (Lyon et al. 2017), are recruited to the site. This structure has been carefully examined in recent years (Du et al. 2013, Lyon et al. 2015, 2017), and believed to serve as an endocytic device to support protein endocytosis and recycling (Vogl et al. 2013, 2014), so that internalized ES proteins can be used to assemble new ES during junction formation/restructuring in stage VIII of the epithelial cycle.

As previously discussed, SFK localization and function require endocytic trafficking. This, in turn, modulates the endocytic events. SFK has been shown to regulate the trafficking/internalization of receptor tyrosine kinases (RTKs) such as FGFR (fibroblast growth factor receptor) and EGFR (epidermal growth factor receptor), as well as integrin-mediated adhesion complexes. SFK targets and phosphorylates components of the endocytic machinery such as dynamin, Eps8, clathrin and clathrinassociated adaptor complex AP2 (assembly polypeptide 2) (Sandilands \& Frame 2008, Sato et al. 2009, Reinecke \& Caplan 2014, Malaga-Trillo \& Ochs 2016). Endocytic internalization of junctional protein complexes can also solicit SFK activity. For instance, during gap junction internalization, Cx43 (connexin 43, a gap junction integral membrane protein) was found to be phosphorylated and associated more with activated SRC. Interaction with and phosphorylation by SFK are critical for endocytosis at tight and adherens junctions between epithelial/endothelial cells as well (Gilleron et al. 2008, Carette et al. 2010, Morton et al. 2013, Reinecke \& Caplan 2014, Chichger et al. 2015, Van Itallie \& Anderson 2018). Nevertheless, the role of SFK on endocytic protein trafficking in the testis was not investigated until recently. Using an in vitro model of primary cultures of Sertoli cells, which is known to mimic much of the Sertoli cell BTB in vivo (Byers et al. 1986, Janecki et al. 1991, 1992, Grima et al. 1992, Nicholls et al. 2009), it was noted that large endocytic vesicles at Sertoli cellcell interface implicating the presence of the basal TBC ultrastructures as reported earlier (Russell 1977a, 1979, Du et al. 2013, Lyon et al. 2015, 2017) were detected in these cultures under electron microscopy (Xiao et al. 2014b). This finding further supports the notion that this in vitro system faithfully reproduces information of the in vivo Sertoli cell BTB. When this in vitro system was used and coupled with biochemical assays to monitor the events of protein endocytosis and recycling, intracellular protein degradation and phagocytosis, it was shown that SRC and YES had differential effects on endocytic vesicle-mediated protein trafficking events (Xiao et al. 2014b). In brief, when knocking down of SRC or YES by RNAi using the corresponding specific siRNA duplexes vs non-targeting negative control siRNA duplexes, SRC knockdown was delayed, whereas YES knockdown enhanced protein endocytosis considerably at the Sertoli cell BTB (Xiao et al. 2014b). Thus, under physiological conditions, SRC and YES promote protein 
endocytosis and protein retention at the Sertoli cellcell interface, respectively. Interestingly, though both SRC and YES knockdown delayed the disappearance of biotinylated/endocytosed proteins (e.g., JAM1, CAR, both are Sertoli cell BTB-associated proteins) from the cell cytosol, YES knockdown decelerated the kinetics of protein recycling back to the Sertoli cell surface (Xiao et al. 2014b), whereas SRC knockdown considerably impeded Sertoli cell phagocytic activity. Taken collectively, these data support the notion that under physiological conditions, YES may be actively involved in protein recycling and SRC in protein degradation and phagocytosis of Sertoli cells. In agreement with this, when TBC is most prominent between late spermatid and Sertoli cell in stage VII tubules, YES has been found at the concave side of spermatid heads in the rat testis which corresponds to the site of apical TBC (Vogl et al. 2014). SRC was strongly associated with residual bodies in stage VIII tubules when its localization was assessed by immunohistochemistry using a specific anti-SRC antibody (Xiao et al. 2014b). These findings thus support the concept that under physiological conditions, SRC promotes protein endocytosis at the ES, but directs the endocytosed proteins to the intracellular endosomemediated degradation pathway. On the other hand, YES also promotes protein endocytosis at the ES, but directs endocytosed proteins to the pathway of protein recycling (perhaps also transcytosis) (Fig. 4). Whether internalization and intracellular sorting of junctional proteins are dependent or independent of SRC/YESmediated tyrosine phosphorylation and whether SRC/YES could function as docking protein in these endocytic trafficking events in the testis have not yet been investigated. But studies from our lab and others have shown that tyrosine phosphorylation of component proteins is essential to the regulation of apical ES and BTB dynamics, and SRC kinase activity is required for germ cell departure from the epithelium (apical
ES disruption) and adhesion of germ cells to Sertoli cells (apical ES assembly) (Zhang et al. 2005, Shupe et al. 2011), implicating that SFK-mediated tyrosine phosphorylation may have a role to play in endocytosis and membrane trafficking during junction restructuring at spermatogenesis.

\section{SRC and YES on F-actin organization in the seminiferous epithelium}

As noted above, both SRC and YES are involved in endocytic vesicle-mediated protein trafficking events, in which SRC promotes endocytosed proteins to enter the endosome-dependent degradation pathway, whereas YES promotes endocytosed proteins to be recycled, illustrating these two SFKs have contrasting roles on the fate of the endocytosed proteins in Sertoli cells during spermatogenesis. While this observation is physiologically important, the underlying biochemical and/or molecular mechanism(s) remains unclear. However, other studies have shown that endocytic vesicle-mediated protein trafficking is a cytoskeletondependent effect, intimately supported by actin- and/ or microtubule-based cytoskeletons (Echarri \& Del Pozo 2015, Kim \& Gadila 2016, West \& Harris 2016), involving Rab GTPases (Progida \& Bakke 2016, Gibieza \& Prekeris 2017, Rout \& Field 2017). Studies have shown that the use of a selective YES inhibitor, SU6656, was found to perturb F-actin organization (Xiao et al. 2011). For instance, treatment of Sertoli cells with SU6656 at $20 \mathrm{nM}$ induced the retraction of actin microfilaments from cell peripheries, with F-actin wrapping around cell nuclei instead of stretching across the entire cell cytosol as in control cells (Xiao et al. 2011). Furthermore, actin microfilaments were extensively truncated following inhibition of YES activity by SU6656 (Xiao et al. 2011), thereby perturbing the ES function. This observation is also consistent with findings that when YES was silenced

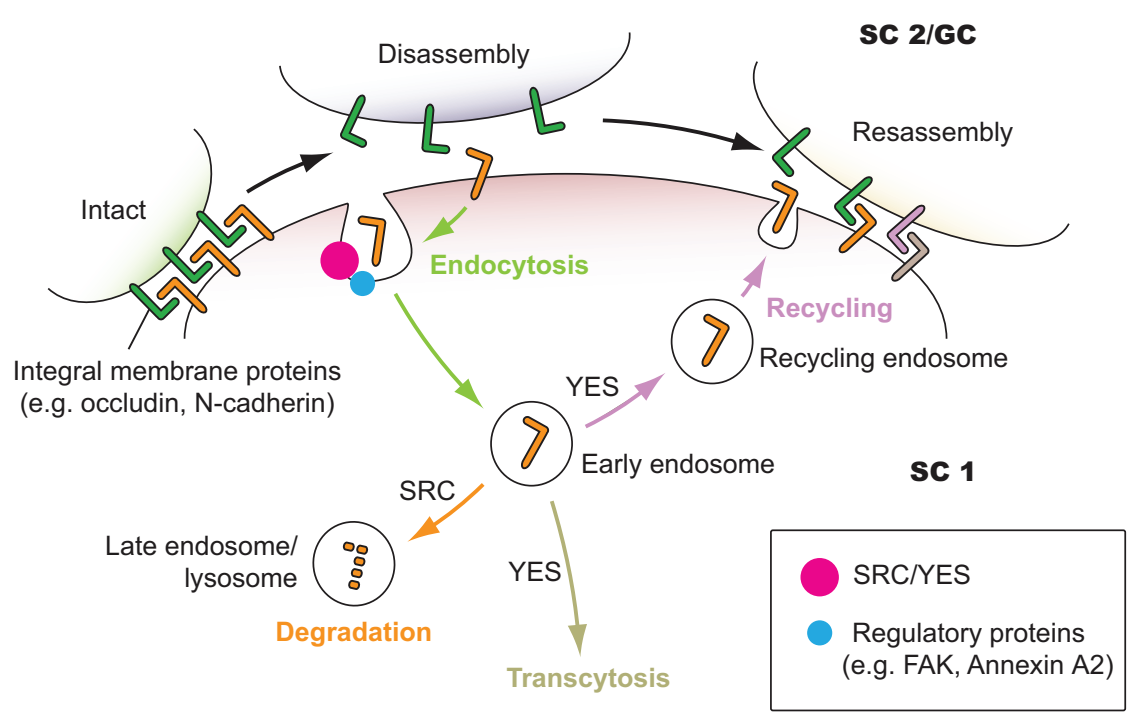

Figure $4 \mathrm{~A}$ hypothetical model illustrating the differential roles of SRC and YES in endocytic vesicle-mediated protein trafficking events at the Sertoli-Sertoli cell (SC) and/or Sertoligerm cell (GC) interface. The figure progresses through representative junction restructuring events from disassembly of junctions on the left to reassembly of junctions on the right. As described in text, SRC is involved in targeting endocytosed proteins for their eventual endosome-mediated degradation, whereas YES is involved in targeting endocytosed proteins for recycling and transcytosis. 
by $\mathrm{RNAi}$, actin microfilaments were disorganized and extensively truncated (Xiao et al. 2014b). This pattern of F-actin disruption is somewhat similar to the knockdown of SRC in Sertoli cells (Xiao et al. 2014b). Collectively, these findings illustrate that both SRC and YES are important regulators of F-actin under physiological conditions by promoting F-actin organization in Sertoli cells to support ES integrity and function. Changes in the organization of F-actin by SRC vs YES, presumably via their action on actin-regulating proteins and/or adhesion protein complexes, facilitate the transport of endocytic vesicles through the degradation vs recycling pathway, respectively. This possibility, while requiring additional future studies in the testis, is in fact supported by studies in other cells and tissues. For instance, mutations or deletions of SFKs in macrophages led to a considerable reduction in cytoskeletal dynamics, impeding cell polarization, migration and phagocytic activity (Baruzzi et al. 2008). On the other hand, SRC (but not FYN) also promotes proper spindle orientation to support prometaphase (Nakayama et al. 2012). In summary, additional studies are needed to better understand how SFKs modulate protein trafficking events through their regulatory role on actin-based cytoskeletons. Furthermore, SRC, FYN and LCK were shown to bind to the microtubule cytoskeleton components such as Tau and A-Tubulin (Klein et al. 2002, Lee 2005), but little is known in the testis. Thus, besides F-actin, future studies should also include analysis of the MT- and vimentinbased cytoskeletons to examine whether SFK modulates MT- and vimentin-based cytoskeletal organization.

\section{Concluding remarks and future perspectives}

There is emerging evidence to support the concept that SFKs regulate spermatogenesis through the differential effects of different SFK members on endocytic vesiclemediated trafficking and F-actin organization in Sertoli cells. However, two questions remain unaddressed. First, what is the overall upstream regulator(s) of SRC and YES on Sertoli cell protein trafficking function? Does this involve specific types of germ cells or other regulators, such as testosterone, cytokines and others? Second, besides SFK members, are there other nonreceptor protein kinases also taking part in these events, such as FAK (focal adhesion kinase)? This is important and studies have shown that FAK and its phosphorylated forms are known regulators of Sertoli cell BTB dynamics (Lie et al. 2012). On the other hand, due to the presence of several $\mathrm{SH}$ domains (Fig. 2) along the polypeptide sequence of SFKs, each member of SFK, such as SRC and YES, can interact with other proteins in Sertoli cells and can even recruit additional partner proteins to widen the scope of its function in the testis. For instance, it is known that SRC is an integrated component of the Cx43 and ZO-1 complex in Sertoli cells in the testis (Gilleron et al. 2008, Li et al. 2009). SFKs are also known regulators to modulate spermatogonial stem cell proliferation and function (Braydich-Stolle et al. 2007), and FYN kinase is involved in GDNF signaling to modulate spermatogonial stem cell function (BraydichStolle et al. 2010). Furthermore, SRC is the downstream protein kinase of non-classical testosterone signaling pathway, shown to be involved in the release of sperm at spermiation (Shupe et al. 2011). It is expected that with recent advances in cell and molecular biology techniques, many of these questions will be answered within the next decade.

\section{Declaration of interest}

The authors declare that there is no conflict of interest that could be perceived as prejudicing the impartiality of this review.

\section{Funding}

This work was supported in parts by grants from the National Natural Science Foundation of China (NSFC) (grant numbers 31371176 to $X X, 81771647,81571426$ and 81170554 to $Y$ N); Qianjiang Talents Program QJD1502029 to X X; Zhejiang Province Department of Science Technology Funding 2016F10010 to X X; the National Institutes of Health, NICHD R01 HD056034 to C Y C; U54 HD029990 Project 5 to CY C.

\section{References}

Amata I, Maffei M \& Pons M 2014 Phosphorylation of unique domains of Src family kinases. Frontiers in Genetics 5 181. (https://doi.org/10.3389/ fgene.2014.00181)

Anguita E \& Villalobo A 2017 Src-family tyrosine kinases and the Ca2+ signal. Biochimica et Biophysica Acta: Molecular Cell Research 1864 915-932. (https://doi.org/10.1016/j.bbamcr.2016.10.022)

Arbesu M, Maffei M, Cordeiro TN, Teixeira JM, Perez Y, Bernado P, Roche S \& Pons M 2017 The unique domain forms a fuzzy intramolecular complex in src family kinases. Structure 25 630.e634-640.e634. (https:// doi.org/10.1016/j.str.2017.02.011)

Banks DB, Chan GN, Evans RA, Miller DS \& Cannon RE 2018 Lysophosphatidic acid and amitriptyline signal through LPA1R to reduce P-glycoprotein transport at the blood-brain barrier. Journal of Cerebral Blood Flow and Metabolism 38 857-868. (https://doi.org/10.1177/027 1678X17705786)

Baruzzi A, Caveggion E \& Berton G 2008 Regulation of phagocyte migration and recruitment by Src-family kinases. Cellular and Molecular Life Sciences 65 2175-2190. (https://doi.org/10.1007/s00018-0088005-6)

Bellve AR, Cavicchia JC, Millette CF, O'Brien DA, Bhatnagar YM \& Dym M 1977 Spermatogenic cells of the prepuberal mouse. Isolation and morphological characterization. Journal of Cell Biology 74 68-85. (https://doi.org/10.1083/jcb.74.1.68)

Bordeleau LJ \& Leclerc P 2008 Expression of hck-tr, a truncated form of the src-related tyrosine kinase hck, in bovine spermatozoa and testis. Molecular Reproduction and Development 75 828-837. (https://doi. org/10.1002/mrd.20814)

Braydich-Stolle L, Kostereva N, Dym M \& Hofmann MC 2007 Role of Src family kinases and N-Myc in spermatogonial stem cell proliferation. Developmental Biology $304 \quad 34-45 . \quad$ (https://doi.org/10.1016/j. ydbio.2006.12.013)

Braydich-Stolle LK, Lucas B, Schrand A, Murdock RC, Lee T, Schlager JJ, Hussain SM \& Hoffmann MC 2010 Silver nanoparticles disrupt GDNF/ Fyn kinase signaling in spermatogonial stem cells. Toxicological Sciences 116 577-589. (https://doi.org/10.1093/toxsci/kfq148) 
Brown MT \& Cooper JA 1996 Regulation, substrates, and functions of Src. Biochimica and Biophysica Acta 1287 121-149. (https://doi. org/10.1016/0304-419X(96)00003-0)

Byers SW, Hadley MA, Djakiew D \& Dym M 1986 Growth and characterization of epididymal epithelial cells and Sertoli cells in dual environment culture chambers. Journal of Andrology 7 59-68. (https:// doi.org/10.1002/j.1939-4640.1986.tb00871.x)

Carette D, Gilleron J, Segretain D \& Pointis G 2010 Heteromeric connexin 43/connexin 33 complex endocytosis: a connexin phosphorylation independent mechanism. Biochimie 92 555-559. (https://doi. org/10.1016/j.biochi.2010.02.003)

Castoria G, Auricchio F \& Migliaccio A 2017 Extranuclear partners of androgen receptor: at the crossroads of proliferation, migration, and neuritogenesis. FASEB Journal 31 1289-1300. (https://doi.org/10.1096/ fj.201601047R)

Cheng CY \& Mruk DD 2002 Cell junction dynamics in the testis: Sertoligerm cell interactions and male contraceptive development. Physiological Reviews 82 825-874. (https://doi.org/10.1152/physrev.00009.2002)

Cheng CY \& Mruk DD 2010 A local autocrine axis in the testes that regulates spermatogenesis. Nature Reviews Endocrinology 6 380-395. (https://doi.org/10.1038/nrendo.2010.71)

Cheng CY \& Mruk DD 2012 The blood-testis barrier and its implications for male contraception. Pharmacological Reviews 64 16-64. (https://doi. org/10.1124/pr.110.002790)

Cheng CY \& Mruk DD 2015 Biochemistry of Sertoli cell/germ cell junctions, germ cell transport, and spermiation in the seminiferous epithelium. In Sertoli Cell Biology, 2nd ed., pp 333-383. Ed MD Griswold. Amsterdam: Elsevier.

Chichger H, Duong H, Braza J \& Harrington EO 2015 p18, a novel adaptor protein, regulates pulmonary endothelial barrier function via enhanced endocytic recycling of VE-cadherin. FASEB Journal 29 868-881. (https:// doi.org/10.1096/fj.14-257212)

Chojnacka K \& Mruk DD 2015 The Src non-receptor tyrosine kinase paradigm: new insights into mammalian Sertoli cell biology. Molecular and Cellular Endocrinology 415 133-142. (https://doi.org/10.1016/j. mce.2015.08.012)

Chu PH, Tsygankov D, Berginski ME, Dagliyan O, Gomez SM, Elston TC, Karginov AV \& Hahn KM 2014 Engineered kinase activation reveals unique morphodynamic phenotypes and associated trafficking for Src family isoforms. PNAS 111 12420-12425. (https://doi.org/10.1073/ pnas.1404487111)

Clermont Y 1972 Kinetics of spermatogenesis in mammals: seminiferous epithelium cycle and spermatogonial renewal. Physiological Reviews 52 198-236. (https://doi.org/10.1152/physrev.1972.52.1.198)

Clermont Y \& Perry B 1957 Quantitative study of the cell population of the seminiferous tubules in immature rats. American Journal of Anatomy $\mathbf{1 0 0}$ 241-267. (https://doi.org/10.1002/aja.1001000205)

Coiras M, Ambrosioni J, Cervantes F, Miró JM \& Alcamí J 2017 Tyrosine kinase inhibitors: potential use and safety considerations in HIV-1 infection. Expert Opinion on Drug Safety 16 547-559. (https://doi.org/1 $0.1080 / 14740338.2017 .1313224)$

de Kretser DM \& Kerr JB 1988 The cytology of the testis. In The Physiology of Reproduction, vol. 1, pp 837-932. Eds E Knobil, JB Neill, LL Ewing, GS Greenwald, CL Markert \& DW Pfaff. New York: Raven Press.

Du M, Young J, De Asis M, Cipollone J, Roskelley C, Takai Y, Nicholls PK, Stanton PG, Deng W, Finlay BB et al. 2013 A novel subcellular machine contributes to basal junction remodeling in the seminiferous epithelium. Biology of Reproduction 88 60. (https://doi.org/10.1095/ biolreprod.112.104851)

Echarri A \& Del Pozo MA 2015 Caveolae - mechanosensitive membrane invaginations linked to actin filaments. Journal of Cell Science $\mathbf{1 2 8}$ 2747-2758. (https://doi.org/10.1242/jcs.153940)

Engen JR, Wales TE, Hochrein JM, Meyn MA, 3rd, Banu Ozkan S, Bahar I \& Smithgall TE 2008 Structure and dynamic regulation of Src-family kinases. Cellular and Molecular Life Sciences 65 3058-3073. (https://doi. org/10.1007/s00018-008-8122-2)

Espada J \& Martin-Perez J 2017 An update on Src family of nonreceptor tyrosine kinases biology. International Review of Cell and Molecular Biology 331 83-122. (https://doi.org/10.1016/bs.ircmb.2016.09.009)

Gaytan F, Lucena MC, Munoz E \& Paniagua R 1986 Morphometric aspects of rat testis development. Journal of Anatomy 145 155-159.
Gibieza P \& Prekeris R 2017 Rab GTPases and cell division. Small GTRPases 9 107-115. (https://doi.org/10.1080/21541248.2017.1313182)

Gilleron J, Fiorini C, Carette D, Avondet C, Falk MM, Segretain D \& Pointis G 2008 Molecular reorganization of Cx43, Zo-1 and SrC complexes during the endocytosis of gap junction plaques in response to a non-genomic carcinogen. Journal of Cell Science 121 4069-4078. (https://doi.org/10.1242/jcs.033373)

Goupil S, La Salle S, Trasler JM, Bordeleau LJ \& Leclerc P 2011 Developmental expression of SRC-related tyrosine kinases in the mouse testis. Journal of Andrology 32 95-110. (https://doi.org/10.2164/ jandrol.110.010462)

Grima J, Pineau C, Bardin CW \& Cheng CY 1992 Rat Sertoli cell clusterin, $\alpha_{2}$-macroglobulin, and testins: biosynthesis and differential regulation by germ cells. Molecular and Cellular Endocrinology 89 127-140. (https:// doi.org/10.1016/0303-7207(92)90219-V)

Hermo L, Pelletier RM, Cyr DG \& Smith CE 2010a Surfing the wave, cycle, life history, and genes/proteins expressed by testicular germ cells. Part 1: background to spermatogenesis, spermatogonia, and spermatocytes. Microscopy Research and Technique 73 241-278. (https://doi. org/10.1002/jemt.20783)

Hermo L, Pelletier RM, Cyr DG \& Smith CE $2010 b$ Surfing the wave, cycle, life history, and genes/proteins expressed by testicular germ cells. Part 5: intercellular junctions and contacts between germ cells and Sertoli cells and their regulatory interactions, testicular cholesterol, and genes/proteins associated with more than one germ cell generation. Microscopy Research and Technique 73 409-494. (https://doi. org/10.1002/jemt.20786)

Hess RA \& de Franca LR 2008 Spermatogenesis and cycle of the seminiferous epithelium. Advances in Experimental Medicine and Biology 636 1-15. (https://doi.org/10.1007/978-0-387-09597-4_1)

Janecki A, Jakubowiak A \& Steinberger A 1991 Regulation of transepithelial electrical resistance in two-compartment Sertoli cell cultures: in vitro model of the blood-testis barrier. Endocrinology 129 1489-1496. (https://doi.org/10.1210/endo-129-3-1489)

Janecki A, Jakubowiak A \& Steinberger A 1992 Effect of cadmium chloride on transepithelial electrical resistance of Sertoli cell monolayers in twocompartment cultures - a new model for toxicological investigations of the 'blood-testis' barrier in vitro. Toxicology and Applied Pharmacology 112 51-57. (https://doi.org/10.1016/0041-008X(92)90278-Z)

Johnson L, Petty CS \& Neaves WB 1980 A comparative study of daily sperm production and testicular composition in humans and rats. Biology of Reproduction 22 1233-1243. (https://doi.org/10.1093/ biolreprod/22.5.1233)

Johnson L, Petty CS \& Neaves WB 1983 Further quantification of human spermatogenesis: germ cell loss during postprophase of meiosis and its relationship to daily sperm production. Biology of Reproduction 29 207-215. (https://doi.org/10.1095/biolreprod29.1.207)

Kasahara K, Nakayama Y, Ikeda K, Fukushima Y, Matsuda D, Horimoto S \& Yamaguchi N 2004 Trafficking of Lyn through the Golgi caveolin involves the charged resides on $\mathrm{aE}$ and a1 herlices in the kinase domain. Journal of Cell Biology 165 641-652. (https://doi.org/10.1083/jcb.200403011)

Kasahara K, Nakayama Y, Kihara A, Matsuda D, Idkeda K, Kuga T, Fukumoto Y, Igarashi Y \& Yamaguchi N 2007 Rapid traffikcing of C-Src, a non-palmitoylated Src-fmaily kinase, between the plasma membrane and late endosomes/lysosomes. Experimental Cell Research 313 2651-2666. (https://doi.org/10.1016/j.yexcr.2007.05.001)

Kierszenbaum AL 2006 Tyrosine protein kinases and spermatogenesis: truncation matters. Molecular Reproduction and Development 73 399-403. (https://doi.org/10.1002/mrd.20456)

Kierszenbaum AL, Rivkin E, Talmor-Cohen A, Shalgi R \& Tres LL 2009 Expression of full-length and truncated Fyn tyrosine kinase transcripts and encoded proteins during spermatogenesis and localization during acrosome biogenesis and fertilization. Molecular Reproduction and Development 76 832-843. (https://doi.org/10.1002/mrd.21049)

Kim K \& Gadila SKG 2016 Cargo trafficking from the trans-Golgi network towards the endosome. Biology of the Cell 108 205-218. (https://doi. org/10.1111/boc.201600001)

Kim CG, Lee H, Gupta N, Ramachandran S, Kaushik I, Srivastava S, Kim SH \& Srivastava SK 2017 Role of forkhead box class o proteins in cancer progression and metastasis. Seminars in Cancer Biology 50 142-151. (https://doi.org/10.1016/j.semcancer.2017.07.007) 
Klein DM \& Cherrington NJ 2014 Organic and inorganic transporters of the testis: a review. Spermatogenesis 4 e979653. (https://doi.org/10.416 $1 / 21565562.2014 .979653)$

Klein C, Kramer EM, Cardine AM, Schraven B, Brandt R \& Trotter J 2002 Process outgrowth of oligodendrocytes is promoted by interaction of fyn kinase with the cytoskeletal protein tau. Journal of Neuroscience 22 698-707. (https://doi.org/10.1523/JNEUROSCI.22-03-00698.2002)

Lalancette C, Bordeleau LJ, Faure RL \& Leclerc P 2006 Bull testicular haploid germ cells express a messenger encoding for a truncated form of the protein tyrosine kinase HCK. Molecular Reproduction and Development 73 520-530. (https://doi.org/10.1002/mrd.20422)

Lee G 2005 Tau and src family tyrosine kinases. Biochimica and Biophysica Acta 1739 323-330. (https://doi.org/10.1016/j.bbadis.2004.09.002)

Lee NPY \& Cheng CY 2005 Protein kinases and adherens junction dynamics in the seminiferous epithelium of the rat testis. Journal of Cellular Physiology 202 344-360. (https://doi.org/10.1002/jcp.20119)

Li MWM, Mruk DD, Lee WM \& Cheng CY 2009 Connexin 43 and plakophilin-2 as a protein complex that regulates blood-testis barrier dynamics. PNAS 106 10213-10218. (https://doi.org/10.1073/ pnas.0901700106)

Lie PPY, Mruk DD, Lee WM \& Cheng CY 2009 Epidermal growth factor receptor pathway substrate 8 (Eps8) is a novel regulator of cell adhesion and the blood-testis barrier integrity in the seminiferous epithelium. FASEB Journal 23 2555-2567. (https://doi.org/10.1096/fj.06-070573)

Lie PPY, Chan AYN, Mruk DD, Lee WM \& Cheng CY 2010 Restricted Arp3 expression in the testis prevents blood-testis barrier disruption during junction restructuring at spermatogenesis. PNAS 107 11411-11416. (https://doi.org/10.1073/pnas.1001823107)

Lie PPY, Mruk DD, Mok KW, Su L, Lee WM \& Cheng CY 2012 Focal adhesion kinase-Tyr ${ }^{407}$ and -Tyr ${ }^{397}$ exhibit antagonistic effects on bloodtestis barrier dynamics in the rat. PNAS 109 12562-12567. (https://doi. org/10.1073/pnas.1202316109)

Lyon KR, Bosseboeuf E \& Vogl AW 2015 An alternative model of tubulobulbar complex internalization during junction remodeling in the seminiferous epithelium of the rat testis. Biology of Reproduction 9312. (https://doi.org/10.1095/biolreprod.115.128942)

Lyon K, Adams A, Piva M, Asghari P, Moore ED \& Vogl AW 2017 Ca2+ signaling machinery is present at intercellular junctions and structures associated with junction turnover in rat Sertoli cells. Biology of Reproduction 96 1288-1302. (https://doi.org/10.1093/biolre/iox042)

Maekawa M, Toyama Y, Yasuda M, Yagi T \& Yuasa S 2002 Fyn tyrosine kinase in Sertoli cells is involved in mouse spermatogenesis. Biology of Reproduction 66 211-221. (https://doi.org/10.1095/biolreprod66.1.211)

Malaga-Trillo E \& Ochs K 2016 Uncontrolled SFK-mediated protein trafficking in prion and Alzheimer's disease. Prion 10 352-361. (https:// doi.org/10.1080/19336896.2016.1221873)

Morton PE, Hicks A, Nastos T, Santis G \& Parsons M 2013 CAR regulates epithelial cell junction stability through control of E-cadherin trafficking. Scientific Reports 3 2889. (https://doi.org/10.1038/srep02889)

Mruk DD \& Cheng CY 2004 Cell-cell interactions at the ectoplasmic specialization in the testis. Trends in Endocrinology and Metabolism 15 439-447. (https://doi.org/10.1016/j.tem.2004.09.009)

Mruk DD, Bonanomi M \& Silvestrini B 2017 Lonidamine-ethyl estermediated remodelling of the Sertoli cell cytoskeleton induces phosphorylation of plakoglobin and promotes its interaction with alpha-catenin at the blood-testis barrier. Reproduction, Fertility, and Development 29 998-1011. (https://doi.org/10.1071/RD15378)

Nakayama Y, Matsui Y, Takeda Y, Okamoto M, Abe K, Fukumoto Y \& Yamaguchi N 2012 c-Src but not Fyn promotes proper spindle orientation in early prometaphase. Journal of Biological Chemistry 287 24905-24915. (https://doi.org/10.1074/jbc.M112.341578)

Nicholls PK, Harrison CA, Gilchrist RB, Farnworth PG \& Stanton PG 2009 Growth differentiation factor 9 is a germ cell regulator of Sertoli cell function. Endocrinology 150 2481-2490. (https://doi.org/10.1210/ en.2008-1048)

Nygaard HB 2018 Targeting Fyn kinase in Alzheimer's disease. Biological Psychiatry $83 \quad 369-376 . \quad$ (https://doi.org/10.1016/j. biopsych.2017.06.004)

O'Donnell L \& O'Bryan MK 2014 Microtubules and spermatogenesis. Seminars in Cell and Developmental Biology 30 45-54. (https://doi. org/10.1016/j.semcdb.2014.01.003)
O'Donnell L, Nicholls PK, O'Bryan MK, McLachlan RI \& Stanton PG 2011 Spermiation: the process of sperm release. Spermatogenesis 1 14-35. (https://doi.org/10.4161/spmg.1.1.14525)

Parvinen M 1982 Regulation of the seminiferous epithelium. Endocrine Reviews 3 404-417. (https://doi.org/10.1210/edrv-3-4-404)

Pelletier RM 2011 The blood-testis barrier: the junctional permeability, the proteins and the lipids. Progress in Histochemistry and Cytochemistry 46 49-127. (https://doi.org/10.1016/j.proghi.2011.05.001)

Perez Y, Maffei M, Igea A, Amata I, Gairi M, Nebreda AR, Bernado P \& Pons M 2013 Lipid binding by the unique and SH3 domains of C-SrC suggests a new regulatory mechanism. Scientific Reports 3 1295. (https:// doi.org/10.1038/srep01295)

Progida C \& Bakke O 2016 Bidirectional traffic between the Golgi and the endosomes - machineries and regulation. Journal of Cell Science $\mathbf{1 2 9}$ 3971-3982. (https://doi.org/10.1242/jcs.185702)

Reinecke J \& Caplan S 2014 Endocytosis and the Src family of nonreceptor tyrosine kinases. Biomolecular Concepts 5 143-155. (https:// doi.org/10.1515/bmc-2014-0003)

Roskoski R Jr 2015 Src protein-tyrosine kinase structure, mechanism, and small molecule inhibitors. Pharmacological Research 94 9-25. (https:// doi.org/10.1016/j.phrs.2015.01.003)

Rout MP \& Field MC 2017 The evoluation of organellar coat compolexes and organization of the eukaryotic cell. Annual Review of Biochemistry $\mathbf{8 6}$ 637-657. (https://doi.org/10.1146/annurev-biochem-061516-044643)

Russell LD 1977a Movement of spermatocytes from the basal to the adluminal compartment of the rat testis. American Journal of Anatomy 148 313-328. (https://doi.org/10.1002/aja.1001480303)

Russell LD 1977 b Observations on rat Sertoli ectoplasmic ('junctional') specializations in their association with germ cells of the rat testis. Tissue and Cell 9 475-498. (https://doi.org/10.1016/0040-8166(77)90007-6)

Russell LD 1979 Observations on the inter-relationships of Sertoli cells at the level of the blood-testis barrier: evidence for formation and resorption of Sertoli-Sertoli tubulobulbar complexes during the spermatogenic cycle of the rat. American Journal of Anatomy 155 259-279. (https://doi. org/10.1002/aja.1001550208)

Russell LD \& Peterson RN 1985 Sertoli cell junctions: morphological and functional correlates. International Review of Cytology 94 177-211. (https://doi.org/10.1016/S0074-7696(08)60397-6)

Sandilands E \& Frame MC 2008 Endosomal trafficking of Src tyrosine kinase. Trends in Cell Biology 18 322-329. (https://doi.org/10.1016/j. tcb.2008.05.004)

Sato I, Obata Y, Kasahara K, Nakayama Y, Fukumoto Y, Yamasaki T, Yokoyama KK, Saito T \& Yamaguchi N 2009 Differential trafficking of Src, Lyn, Yes and Fyn is specified by the state of palmitoylation in the $\mathrm{SH} 4$ domain. Journal of Cell Science 122 965-975. (https://doi.org/10.1242/ jcs.034843)

Sharpe RM, McKinnell C, Kivlin C \& Fisher JS 2003 Proliferation and functional maturation of Sertoli cells, and their relevance to disorders of testis function in adulthood. Reproduction 125 769-784. (https://doi. org/10.1530/rep.0.1250769)

Shupe J, Cheng J, Puri P, Kostereva N \& Walker WH 2011 Regulation of Sertoli-germ cell adhesion and sperm release by FSH and nonclassical testosterone signaling. Molecular Endocrinology 25 238-252. (https:// doi.org/10.1210/me.2010-0030)

Singh DK, Deshmukh RK, Narayanan PK, Shivaji S \& Siva AB 2017 SRC family kinases in hamster spermatozoa: evidence for the presence of LCK. Reproduction 153 655-669. (https://doi.org/10.1530/REP-160591)

Smith BE \& Braun RE 2012 Germ cell migration across Sertoli cell tight junctions. Science 338 798-802. (https://doi.org/10.1126/ science.1219969)

Sprowl JA, Ong SS, Gibson AA, Hu S, Du G, Lin W, Li L, Bharill S, Ness RA, Stecula A et al. 2016 A phosphotyrosine switch regulates organic cation transporters. Nature Communications 7 10880. (https://doi.org/10.1038/ ncomms10880)

Stein PL, Vogel H \& Soriano P 1994 Combined deficiencies of Src, Fyn, and Yes tyrosine kinases in mutant mice. Genes and Development 8 1999-2007. (https://doi.org/10.1101/gad.8.17.1999)

Takeda H, Kawamura Y, Miura A, Mori M, Wakamatsu A, Yamamoto J, Isogai T, Matsumoto M, Nakayama KI, Natsume T et al. 2010 Comparative analysis of human SRC-family kinase substrate specificity 
in vitro. Journal of Proteome Research 9 5982-5993. (https://doi. org/10.1021/pr100773t)

Tang EI, Mruk DD \& Cheng CY 2016 Regulation of microtubule (MT)-based cytoskeleton in the seminiferous epithelium during spermatogenesis. Seminars in Cell and Developmental Biology 59 35-45. (https://doi. org/10.1016/j.semcdb.2016.01.004)

Thomas SM \& Brugge JS 1997 Cellular functions regulated by Src family kinases. Annual Review of Cell and Developmental Biology 13 513-609. (https://doi.org/10.1146/annurev.cellbio.13.1.513)

Van Itallie CM \& Anderson JM 2018 Phosphorylation of tight junction transmembrane proteins: many sites, much to do. Tissue Barriers 6 e1382671. (https://doi.org/10.1080/21688370.2017.1382671)

Vogl A, Pfeiffer D, Redenbach D \& Grove B 1993 Sertoli cell cytoskeleton. In The Sertoli Cell, pp 39-86. Eds L Russell \& M Griswold. Clearwater: Cache River Press.

Vogl AW, Pfeiffer DC, Mulholland D, Kimel G \& Guttman J 2000 Unique and multifunctional adhesion junctions in the testis: ectoplasmic specializations. Archives of Histology and Cytology 63 1-15. (https:// doi.org/10.1679/aohc.63.1)

Vogl AW, Vaid KS \& Guttman JA 2008 The Sertoli cell cytoskeleton. Advances in Experimental Medicine and Biology 636 186-211. (https:// doi.org/10.1007/978-0-387-09597-4_11)

Vogl AW, Young JS \& Du M 2013 New insights into roles of tubulobulbar complexes in sperm release and turnover of blood-testis barrier. International Review of Cell and Molecular Biology 303 319-355. (https://doi.org/10.1016/B978-0-12-407697-6.00008-8)

Vogl AW, Du M, Wang XY \& Young JS 2014 Novel clathrin/actinbased endocytic machinery associated with junction turnover in the seminiferous epithelium. Seminars in Cell and Developmental Biology 30 55-64. (https://doi.org/10.1016/j.semcdb.2013.11.002)

Wen Q, Tang EI, Xiao X, Gao Y, Chu DS, Mruk DD, Silvestrini B \& Cheng CY 2016 Transport of germ cells across the seminiferous epithelium during spermatogenesis-the involvement of both actin- and microtubule-based cytoskeletons. Tissue Barriers 4 e1265042. (https://doi.org/10.1080/216 88370.2016.1265042)

West JJ \& Harris TJ 2016 Cadherin trafficking for tissue morphogenesis: control and consequences. Traffic 17 1233-1243. (https://doi. org/10.1111/tra.12407)

Xiao X, Mruk DD, Lee WM \& Cheng CY 2011 c-Yes regulates cell adhesion at the blood-testis barrier and the apical ectoplasmic specialization in the seminiferous epithelium of rat testes. International Journal of Biochemistry and Cell Biology 43 651-665. (https://doi.org/10.1016/j. biocel.2011.01.008)

Xiao X, Mruk DD, Cheng FL \& Cheng CY 2012 c-Src and c-yes are two unlikely partners of spermatogenesis and their roles in blood-testis barrier dynamics. Advances in Experimental Medicine and Biology 763 295-317.
Xiao X, Mruk DD \& Cheng CY 2013 c-Yes regulates cell adhesion at the apical ectoplasmic specialization-blood-testis barrier axis via its effects on protein recruitment and distribution. American Journal of Physiology: Endocrinology and Metabolism 304 E145-E159. (https://doi. org/10.1152/ajpendo.00422.2012)

Xiao X, Mruk DD, Wong CKC \& Cheng CY 2014a Germ cell transport across the seminiferous epithelium during spermatogenesis. Physiology 29 286-298. (https://doi.org/10.1152/physiol.00001.2014)

Xiao X, Mruk DD, Wong EWP, Lee WM, Han D, Wong CKC \& Cheng CY $2014 b$ Differential effects of c-Src and c-yes on the endocytic vesiclemediated trafficking events at the Sertoli cell blood-testis barrier: an in vitro study. American Journal of Physiology: Endocrinology and Metabolism 307 E553-E562. (https://doi.org/10.1152/ajpendo.00176.2014)

Young JS \& Vogl AW 2012 Focal adhesion proteins zyxin and vinculin are co-distributed at tubulobulbar complexes. Spermatogenesis 2 63-68. (https://doi.org/10.4161/spmg.19391)

Young JS, Guttman JA, Vaid KS, Shahinian H \& Vogl AW 2009a Cortactin (CTTN), N-WASP (WASL), and clathrin (CLTC) are present at podosomelike tubulobulbar complexes in the rat testis. Biology of Reproduction $\mathbf{8 0}$ 153-161. (https://doi.org/10.1095/biolreprod.108.070615)

Young JS, Guttman JA, Vaid KS \& Vogl AW 2009b Tubulobulbar complexes are intercellular podosome-like structures that internalize intact intercellular junctions during epithelial remodeling events in the rat testis. Biology of Reproduction 80 162-174. (https://doi.org/10.1095/ biolreprod.108.070623)

Young JS, De Asis M, Guttman J \& Vogl AW 2012a Cortactin depletion results in short tubulobulbar complexes and spermiation failure in rat testes. Biology Open 1 1069-1077. (https://doi.org/10.1242/bio.20122519)

Young JS, Takai Y, Kojic KL \& Vogl AW $2012 b$ Internalization of adhesion junction proteins and their association with recycling endosome marker proteins in rat seminiferous epithelium. Reproduction 143 347-357. (https://doi.org/10.1530/REP-11-0317)

Zhang J, Wong CH, Xia W, Mruk DD, Lee NP, Lee WM \& Cheng CY 2005 Regulation of Sertoli-germ cell adherens junction dynamics via changes in protein-protein interactions of the $\mathrm{N}$-cadherin-beta-catenin protein complex which are possibly mediated by $\mathrm{c}$-Src and myotubularinrelated protein 2: an in vivo study using an androgen suppression model. Endocrinology 146 1268-1284. (https://doi.org/10.1210/en.2004-1194)

Received 25 September 2018

First decision 26 October 2018

Revised manuscript received 3 December 2018

Accepted 3 January 2019 